\title{
A Novel Design Strategy for Suppressing Efficiency Roll-Off of Blue Thermally Activated Delayed Fluorescence Molecules through Donor-Acceptor Interlocking by $\mathrm{C}-\mathrm{C}$ Bonds
}

\author{
Tae Hui Kwon ${ }^{1,+}{ }^{\text {, Soon Ok Jeon }}{ }^{2, *,+} \oplus$, Masaki Numata ${ }^{3}$, Hasup Lee ${ }^{2}$, Yeon Sook Chung ${ }^{2}$, \\ Jong Soo Kim ${ }^{2}$, Soo-Ghang Ihn ${ }^{2} \mathbb{D}$, Myungsun Sim ${ }^{2}$, Sunghan Kim ${ }^{2}$ and Byeong Moon Kim ${ }^{1, *}$ \\ 1 Department of Chemistry, Seoul National University, Seoul 08826, Korea; mkmjkm@snu.ac.kr \\ 2 Samsung Advanced Institute of Technology, Samsung Electronics Co., Ltd., 130 Samsung-ro, Suwon-si, \\ Gyeonggi-do 16678, Korea; hasup.lee@samsung.com (H.L.); ys2.chung@samsung.com (Y.S.C.); \\ js100.kim@samsung.com (J.S.K.); sg.ihn@samsung.com (S.-G.I.); ms84.sim@samsung.com (M.S.); \\ shan0819.kim@samsung.com (S.K.) \\ 3 Samsung R\&D Institute Japan, 2-7 Sugasawa-cho, Tsurumu-ku, Yokohama 230-0027, Japan; \\ mnumata@samsung.com \\ * Correspondence: so.jeon@samsung.com (S.O.J.); kimbm@snu.ac.kr (B.M.K.) \\ + These authors contributed equally to this work.
}

Received: 19 November 2019; Accepted: 3 December 2019; Published: 5 December 2019

\begin{abstract}
The short material lifetime of thermally activated delayed fluorescence (TADF) technology is a major obstacle to the development of economically feasible, highly efficient, and durable devices for commercial applications. TADF devices are also hampered by insufficient operational stability. In this paper, we report the design, synthesis, and evaluation of new TADF molecules possessing a sterically twisted skeleton by interlocking donor and acceptor moieties through a $\mathrm{C}-\mathrm{C}$ bond. Compared to C-N-bond TADF molecules, such as CPT2, the C-C-bond TADF molecules showed a large dihedral angle increase by more than 30 times and a singlet-triplet energy-gap decrease to less than $0.22 \mathrm{eV}$ because of the steric hindrance caused by the direct $\mathrm{C}-\mathrm{C}$ bond connection. With the introduction of a dibenzofuran core structure, devices comprising BMK-T317 and BMK-T318 exhibited a magnificent display performance, especially their external quantum efficiencies, which were as high as $19.9 \%$ and $18.8 \%$, respectively. Moreover, the efficiency roll-off of BMK-T318 improved significantly $(26.7 \%)$. These results indicate that stability of the material can be expected through the reduction of their singlet-triplet splitting and the precise adjustment of dihedral angles between the donor-acceptor skeletons.
\end{abstract}

Keywords: organic light-emitting diode (OLED); thermally activated delayed fluorescence (TADF); blue emitter; C-C bond; synthesis; external quantum efficiency (EQE); efficiency roll-off

\section{Introduction}

Organic light-emitting diodes (OLEDs) are considered powerful components of lighting devices. They have enormous potential applications to general lighting, larger displays, and flexible displays because of their superior color properties, thinness, and flexibility [1-6]. However, traditional OLEDs have limitations, such as low efficiency, because only $25 \%$ of the singlet excitons can be harvested in accordance with quantum mechanics [7-9]. Tireless efforts toward increasing the efficiency and stability of OLEDs have continued, and various photophysical phenomena in the field of OLEDs have been discovered, such as singlet fission, triplet-triplet annihilation, and thermally activated delayed 
fluorescence (TADF) [7]. In particular, TADF emitters allow an internal quantum efficiency theoretically up to $100 \%$ by harvesting both singlet and triplet excitons via reverse intersystem crossing (RISC). Thus, TADF technology has attracted significant attention [10,11].

To facilitate the RISC process of the TADF phenomenon, it is necessary to have a small energy gap between the lowest singlet energy and triplet energy in excited states, which is typically less than $0.37 \mathrm{eV}$ [12-16]. The backbones of the TADF emitters are made up of electron-donating and -accepting moieties for spatial separation of highest occupied molecular orbital (HOMO) and lowest occupied molecular orbital (LUMO) distributions. Weak overlap of HOMO and LUMO tends to lead to small singlet-triplet energy gaps $\left(\Delta E_{\mathrm{ST}}\right)$ [17-19]. Because generally used donor units contain aniline- and carbazole-type electron-rich functional groups, the bridge between the donor and acceptor moiety is usually interlocked by a $\mathrm{C}-\mathrm{N}$ bond [20-29]. The carbazole-type donor moiety can be readily introduced through a $\mathrm{C}-\mathrm{N}$ bond connection to the main building block, typically via nucleophilic aromatic substitution reactions $\left(\mathrm{S}_{\mathrm{N}} \mathrm{Ar}\right)$. The average bond dissociation energy of a $\mathrm{C}-\mathrm{N}$ bond $(305 \mathrm{KJ} / \mathrm{mol})$ is lower than that of a $\mathrm{C}-\mathrm{C}$ bond $(347 \mathrm{KJ} / \mathrm{mol})$; therefore, the $\mathrm{C}-\mathrm{N}$ bonds connected between the donor and acceptor units are considered susceptible to the degradation of TADF emitters during the photoluminescence process. In particular, deep-blue emitters have a large excitation energy, approximately $3.0 \mathrm{eV}$ [11]. To alleviate possible degradation of deep-blue TADF materials, a more stable junction between the donor and acceptor groups is deemed more desirable than the C-N bond connection.

Herein, we demonstrate that a new molecular design, employing a $\mathrm{C}-\mathrm{C}$ bond interlocking donor and acceptor moieties, can lead to the stability improvement of blue TADF emitters. Our modelling studies on a series of $C-C$ bonding TADF emitters suggested lower calculated $\Delta E_{S T}$ values, which was one of the most important factors for increased TADF phenomenon over those of the general $\mathrm{C}-\mathrm{N}$-bond blue TADF emitters (e.g., the $\Delta E_{\mathrm{ST}}$ values of BMK-T138 and CPT2 were 0.10 and $0.32 \mathrm{eV}$, respectively) $[28,29]$. Therefore, we designed and synthesized four new TADF emitters having interlocked acceptor units on their carbazole or dibenzofuran backbone structures via $\mathrm{C}-\mathrm{C}$ bond connections. Between the two backbone structures, those containing the dibenzofuran moiety were characterized by short delayed fluorescence lifetimes $\left(\tau_{\mathrm{DF}}\right)$, owing to high kRISC values. Thus, compounds equipped with the dibenzofuran core structure were expected to be even more stable than those with the carbazole structure. Newly designed TADF emitters consist of an interlocked acceptor at the central core, coupled with a carbazole or dibenzofuran donor in the backbone structure for a small $\Delta E_{\mathrm{ST}}$. Restricted rotation of the central core via a $\mathrm{C}-\mathrm{C}$-bonding architecture leads to a large dihedral angle between the donor and acceptor because of the large steric hindrance, affecting the short delayed fluorescence lifetime $\left(\tau_{\mathrm{DF}}\right)[30]$.

\section{Materials and Methods}

\subsection{General Information}

Chemicals were purchased from Sigma-Aldrich Co. (Yongin, Korea), Tokyo Chemical Industry Co. (Seoul, Korea), and Wako Pure Chemical Industries Ltd. (Seoul, Korea), and they were used without further purification. The organic materials used in this study that did not have precedent syntheses were synthesized and characterized according to the methods described in the Supplementary Information. The ${ }^{1} \mathrm{H}$ and ${ }^{13} \mathrm{C}$ nmR spectra were measured using an Agilent 400-MR DD2 magnetic resonance system $(400 \mathrm{MHz})$ spectrophotometer (Seoul, Korea) or a Bruker ASCEND $500(500 \mathrm{MHz})$ spectrophotometer (Seongnam, Korea). Chemical shifts were measured as parts per million ( $d$ values) from tetramethylsilane as an internal standard at probe temperature in $\mathrm{CD}_{2} \mathrm{Cl}_{2}, \mathrm{CDCl}_{3}$, or DMSO- $\mathrm{D}_{6}$ for neutral compounds. Coupling constants were provided in $\mathrm{Hz}$, with the following spectral pattern designations: $s$, singlet; $d$, doublet; $t$, triplet; $q$, quartet; quint, quintet; $m$, multiplet; br, broad; and app, apparent. The matrix-assisted laser desorption/ionization-time of flight (MALDI-TOF) mass spectra were recorded using a Bruker Ultraflex III TOF/TOF 200 spectrometer (Seongnam, 
Korea). The ultraviolet-visible (UV-vis) spectra were obtained by means of a Varian model UV-vis near-infrared spectrophotometer 5000 (Seoul, Korea), and the fluorescence spectra were measured on a HITACHI F7000 spectrometer (Seoul, Korea) for the solution states. The UV-vis absorption and solution photoluminescence (PL) emission spectra of materials were obtained from a dilute toluene solution $\left(1 \times 10^{-5} \mathrm{M}\right)$, whereas the solid PL spectra were obtained from thin films prepared by vacuum evaporation. Triplet energy values of the TADF materials were obtained from the photoluminescence spectra at (Suwon, Korea) $77 \mathrm{~K}$ using liquid $\mathrm{N}_{2}$.

\subsection{Synthesis}

Synthetic details and spectral analysis data of all products are included in the electronic supplementary information (ESI).

\subsection{Device Fabrication and Measurement}

The organic layers were deposited on precleaned $\operatorname{In}_{2} \mathrm{O}_{5} \mathrm{Sn}$ (ITO) glass substrates through the use of a thermal evaporation system having a vacuum pressure of $<1.0 \times 10^{-6}$ torr. A $1 \mathrm{~nm}$ thick 8-hydroxyquinolinolatolithium (Liq.) and a $100 \mathrm{~nm}$ thick $\mathrm{Al}$ (or Ag) were deposited as a cathode via thermal evaporation. The deposition rates of the organic and metal layers were about 0.1 and $0.5 \mathrm{~nm} \mathrm{~s}^{-1}$, respectively. That of the Liq. layer was about $0.01 \mathrm{~nm} \mathrm{~s}^{-2}$. The active device area of $4 \mathrm{~mm}^{2}$ was defined by the overlapped area of the ITO and Al electrodes. The hole-only device structure was ITO/dipyrazino[2,3-f:2' $3^{\prime}$-h] quinoxaline-2,3,6,7,10,11-hexacarbonitrile (HAT-CN, $10 \mathrm{~nm}$ )/N4,N4'-di(naphthalen-1-yl)-N4,N4'-diphenyl-[1,1'-biphenyl]-4,4'-diamine (NPB, $50 \mathrm{~nm}$ )/TADF material $(30 \mathrm{~nm}) / \mathrm{NPB}(10 \mathrm{~nm}) / \mathrm{Al}$. The device structure was ITO $(50 \mathrm{~nm}) / \mathrm{HAT}-\mathrm{CN}(10 \mathrm{~nm}) / \mathrm{NPB}$ $(50 \mathrm{~nm}) / 1,3-d i(9 H$-carbazol-9-yl)benzene $(\mathrm{mCP}, 10 \mathrm{~nm}) /$ Host:TADF emitter $(30 \mathrm{~nm}, 15 \mathrm{wt} \%) /$ dibenzo[b,d]furan-2,8-diylbis(diphenylphosphine oxide) (DBFPO, $10 \mathrm{~nm}$ )/DBFPO:Liq $(30 \mathrm{~nm}, 50$ $\left.{ }_{\mathrm{wt}} \%\right) / \mathrm{Liq}(1 \mathrm{~nm}) / \mathrm{Al}(200 \mathrm{~nm})$. The hosts were bis[2-(diphenylphosphino)phenyl] ether oxide (DPEPO) and 3',5-di(9H-carbazol-9-yl)-[1,1'-biphenyl]-3-carbonitrile (mCBP-CN), and the TADF emitters were BMK-T138, BMK-T139, BMK-T317, and BMK-T318, respectively. Current, voltage, and luminance of the OLEDs were measured with a Keithley 2400 Source-Meter (Suwon, Korea) and PR-650 spectroradiometer (Suwon, Korea). Lifespan measurement of the OLEDs was performed at a constant current mode.

\section{Results and Discussion}

\subsection{Synthesis}

We selected four compounds among candidate structures suggested from virtual simulation screening. Diphenyltriazine attached at the 2- or 4-position of the backbone structures was selected as an acceptor unit, and a carbazole moiety at the 3-position of the backbone structure was chosen as an additional donor unit. The close-in location of the acceptor and additional donor was chosen for the installment of a proper twist angle between the two units.

As shown in Scheme 1, 3-fluoro-9H-carbazole (1) was selected as a common starting material for the synthesis of BMK-T138 and BMK-T139. After phenylation on the carbazole nitrogen, a boronic acid unit was introduced at the carbazole ring to yield a mixture of isomers $3 \mathrm{a}$ and $3 \mathrm{~b}$ in a 5:1 ratio. Suzuki-Miyaura cross-coupling reaction of the resulting boronic acid derivative with a triazine derivative (4) in the presence of a $\mathrm{Pd}$ catalyst provided a mixture of regioisomers, 2-(4,6-diphenyl-1,3,5-triazin-2-yl)-3-fluoro-9-phenyl-9H-carbazole (5a) and 3-fluoro-4-(4,6-diphenyl-1,3,5-triazin-2-yl)-9-phenyl-9H-carbazole (5b), which were readily separated via column chromatography. The two regioisomers, $5 \mathrm{a}$ and $5 \mathrm{~b}$, were obtained in $36 \%$ and $7 \%$ yields, respectively. Finally, $\mathrm{S}_{\mathrm{N}} \mathrm{Ar}$ reactions of $5 \mathrm{a}$ and $5 \mathrm{~b}$ with a carbazole unit provided the target molecules, BMK-T138 and BMK-T139, in 49\% and 85\% yields, respectively. 


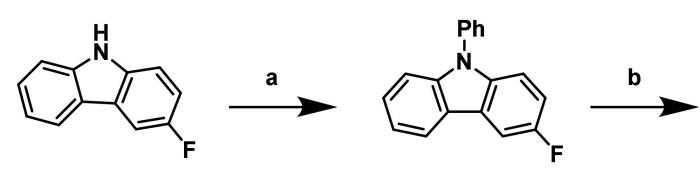

1

2

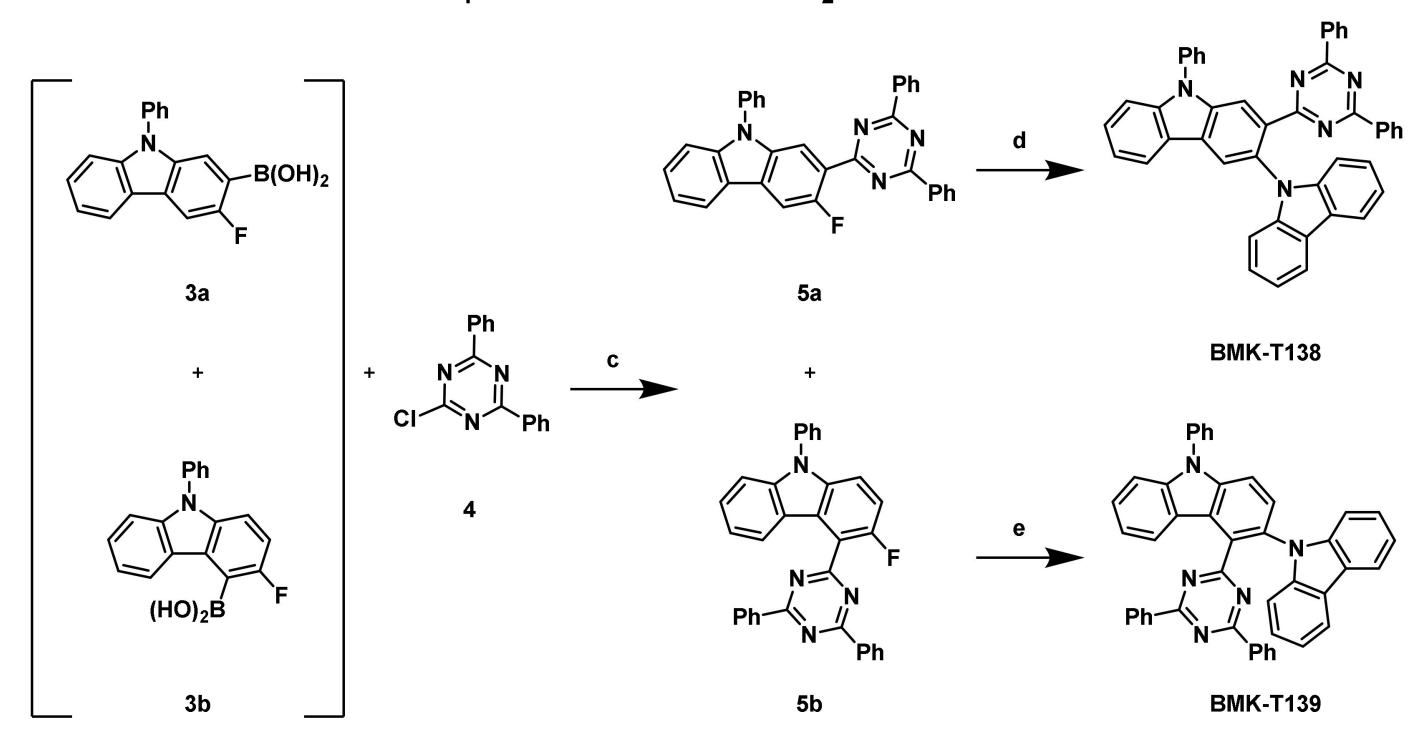

Scheme 1. Synthetic routes of BMK-T138 and BMK-T139: (a) iodobenzene, $\mathrm{K}_{3} \mathrm{PO}_{4}, \mathrm{CuI}$, $N, N^{\prime}$-diacetylcyclohexane-1,2-diamine, dioxane, $80{ }^{\circ} \mathrm{C} ; 86 \%$; (b) triisopropyl borate, lithium 2,2,6,6-tetramethylpiperidide, tetrahydrofuran (THF), $-60{ }^{\circ} \mathrm{C}$, then $1 \mathrm{~N} \mathrm{HCl}$, r.t.; (c) $\mathrm{Pd}(\mathrm{OAc})_{2}$, tri-o-tolyphosphine, $\mathrm{H}_{2} \mathrm{O}$, THF, $80{ }^{\circ} \mathrm{C}, 36 \%$ yield from 2 steps; (d) Carbazole, $t \mathrm{BuOK}$, $\mathrm{N}, \mathrm{N}$-dimethylformamide (DMF), $160{ }^{\circ} \mathrm{C}, 49 \%$; (e) Carbazole, $t \mathrm{BuOK}, \mathrm{DMF}, 160{ }^{\circ} \mathrm{C}, 85 \%$.

For the construction of molecules containing a dibenzofuran moiety instead of a carbazole unit at the backbone structure, a synthetic pathway featuring 3-bromo-2-fluorodibenzo[b,d]furan (6) as a key intermediate molecule was adopted, as shown in Scheme 2. Compound 6 secured the regioselective introduction of the boronic acid moiety for the construction of BMK-T317, which called for adjoining triazole and carbazole units. Compound 6 was purchased from Medigen Co. (Taipei city, Taiwan) and used without further purification. After introducing a carbazole unit at the 3-position of compound 6 via a $S_{N}$ Ar reaction, we converted the bromo group to the corresponding boronic acid (8) via borylation at the 2-bromo position, followed by hydrolysis. A Suzuki-Miyaura cross-coupling reaction was finally carried out with a triazine derivative (4), using a Pd catalyst in the same manner as shown in Scheme 1 to provide the desired compound, BMK-T317, at a 45\% yield.

For the synthesis of BMK-T318, a new synthetic pathway was devised, which allowed the introduction of a carbazole unit before a triazine one, as shown in Scheme 3. The placement of the triazine unit in BMK-T318 required compound 9 as a key intermediate. The dibenzofuran backbone unit (9) was also purchased from Medigen Co. and used without further purification. After introduction of the carbazole unit via the $S_{N}$ Ar reaction at the 3-position of the benzofuran backbone (9), borylation was carried out at the 4-position for the final Suzuki-Miyaura cross-coupling reaction with a triazine unit (4) in the same manner as shown in Scheme 2.

All four final products were purified through flash-column chromatography followed by sublimation to ensure $>99.5 \%$ purities. Detailed spectral data are included in the electronic supplementary information (ESI).

Thermal properties of the C-C bonding blue TADF emitters were characterized by thermogravimetric analysis (TGA) and differential scanning calorimetry (DSC). TGA and DSC curves of the new $\mathrm{C}-\mathrm{C}$ bonding blue TADF emitters are shown in Figure S1 (ESI). Four distinct transitions were seen in the second-heating/-cooling DSC scans of BMK-T138, BMK-T139, BMK-T 317, and BMK-T318. These materials showed glass transition temperatures $\left(T_{\mathrm{g}}\right)$ of $133,130,116$, and $87.9^{\circ} \mathrm{C}$, respectively. 
The decomposition temperatures $\left(T_{\mathrm{d}}\right.$, corresponding to $5 \%$ weight loss) of $\mathrm{C}-\mathrm{C}$ bonding blue TADF emitters were in the range of 325 to $409{ }^{\circ} \mathrm{C}$, which demonstrated their thermal robustness. The fact that apparent complete thermal decomposition with zero\% weight remaining was observed in the TGA curves suggests that the materials could be readily evaporated to form thin films.

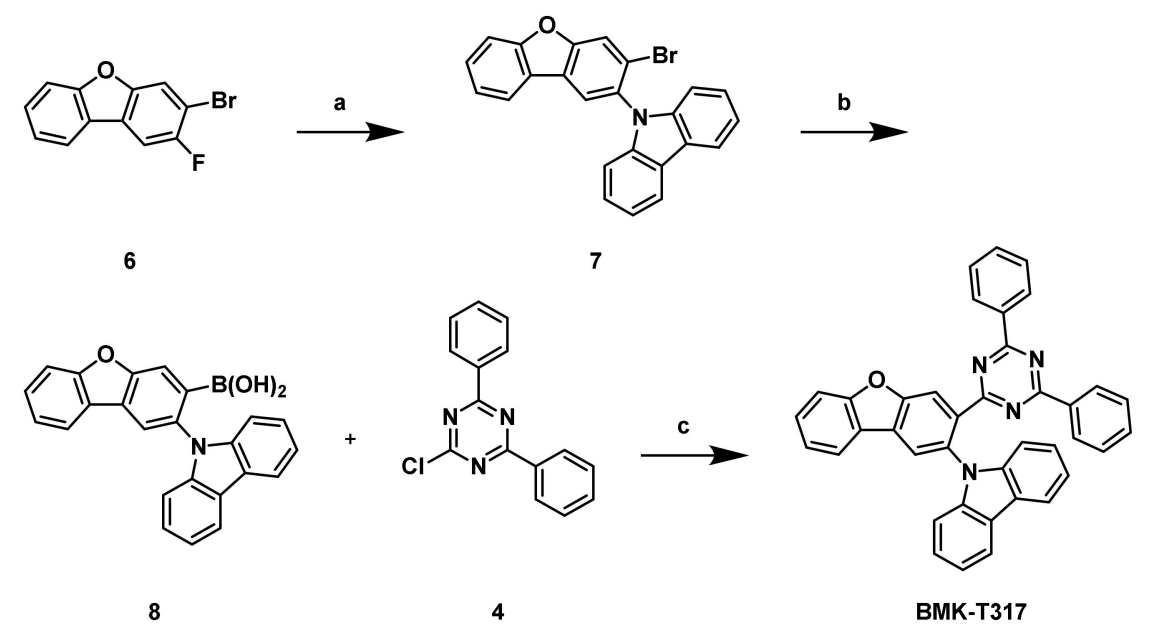

Scheme 2. Synthetic routes of BMK-T317: (a) Carbazole, NaH, $\mathrm{N}, \mathrm{N}$-dimethyl acetamide (DMA), $160{ }^{\circ} \mathrm{C}$, $44 \%$; (b) trimethyl borate, $n$-butyllithium, THF, $-78{ }^{\circ} \mathrm{C}$, then $2 \mathrm{~N} \mathrm{HCl}$, r.t., $51 \%$; (c) $\mathrm{Pd}\left(\mathrm{PPh}_{3}\right)_{4}, 1 \mathrm{M}$ $\mathrm{K}_{2} \mathrm{CO}_{3}$ in $\mathrm{H}_{2} \mathrm{O}, \mathrm{THF}, 80^{\circ} \mathrm{C}, 45 \%$.

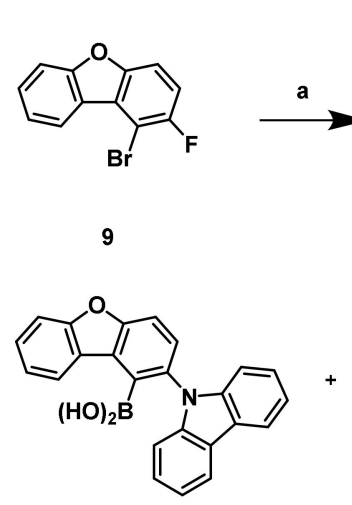

11

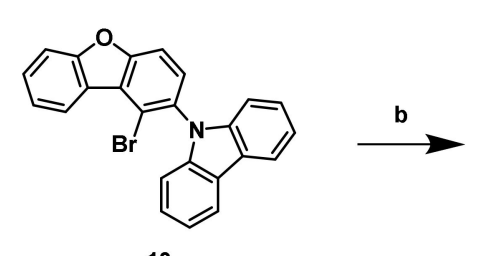

10

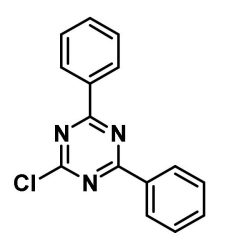

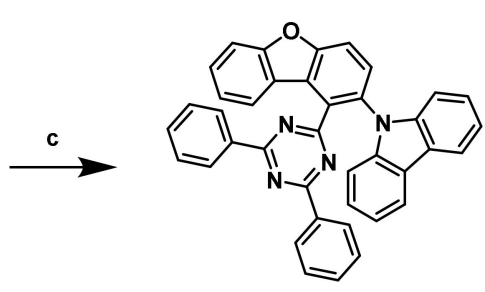

BMK-T318

Scheme 3. Synthetic routes of BMK-T318: (a) Carbazole, $\mathrm{NaH}, \mathrm{DMA}, 160{ }^{\circ} \mathrm{C}, 19 \%$; (b) trimethyl borate, $n$-butyllithium, $\mathrm{THF},-78{ }^{\circ} \mathrm{C}$, then $2 \mathrm{~N} \mathrm{HCl}$, r.t., $55 \%$; (c) $\mathrm{Pd}\left(\mathrm{PPh}_{3}\right)_{4}, 1 \mathrm{M} \mathrm{K}_{2} \mathrm{CO}_{3}$ in $\mathrm{H}_{2} \mathrm{O}, \mathrm{THF}, 80{ }^{\circ} \mathrm{C}$, $53 \%$.

\subsection{Calculation}

The molecular orbital distributions of the emitter molecules are shown in Figure 1. As can be expected from the molecular structures, the HOMOs were localized on the carbazole-donor unit, whereas the LUMOs were dispersed over the diphenyl triazine-acceptor unit. Strengths of donor and acceptor of the materials were affected by their energy levels. Especially, the dibenzofuran core structure tended to be the weak electron transport (ET) type, compared to that of the carbazole core structure. Therefore, the energy band of dibenzofuran-based $C-C$ bond molecules was lower than that of the carbazole-based $\mathrm{C}-\mathrm{C}$ bond molecules to maintain the energy band gap (Table 1). This led to a lower HOMO energy level to balance the exciton in emission layer (EML) and to maintain blue emission. Four TADF emitters interlocking through the $\mathrm{C}-\mathrm{C}$ bonds were equipped with greater dihedral angles, warranting lower $\Delta E_{\mathrm{ST}}$ values than those of TADF emitters interlocking through $\mathrm{C}-\mathrm{N}$ bonds. In these molecules, hindered bond rotation caused by repulsive steric interaction not only partly interrupted the conjugation system, they also caused sectional deformation of both the linear and planar molecular 
structures, which inevitably affected the dihedral angle and emitter characteristics, such as $\Delta E_{\mathrm{ST}}$ and oscillator strength $(f)$. Therefore, the placement of a $\mathrm{C}-\mathrm{C}$ bond on the donor-acceptor connection led to low efficiency roll-off for the newly designed material.
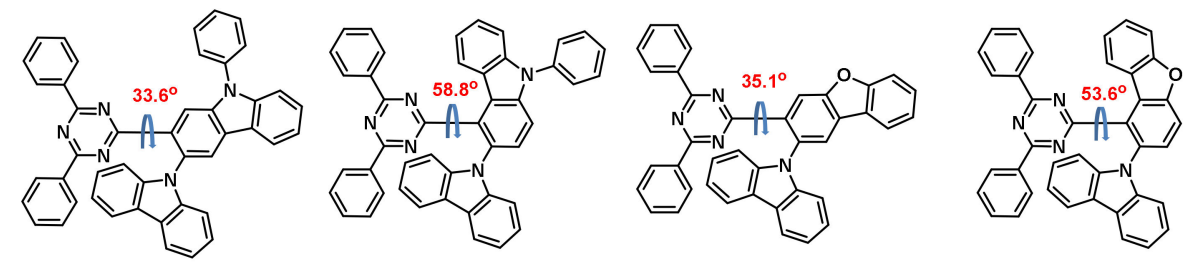

BMK-T138

BMK-T139

BMK-T317

BMK-T318

NTO
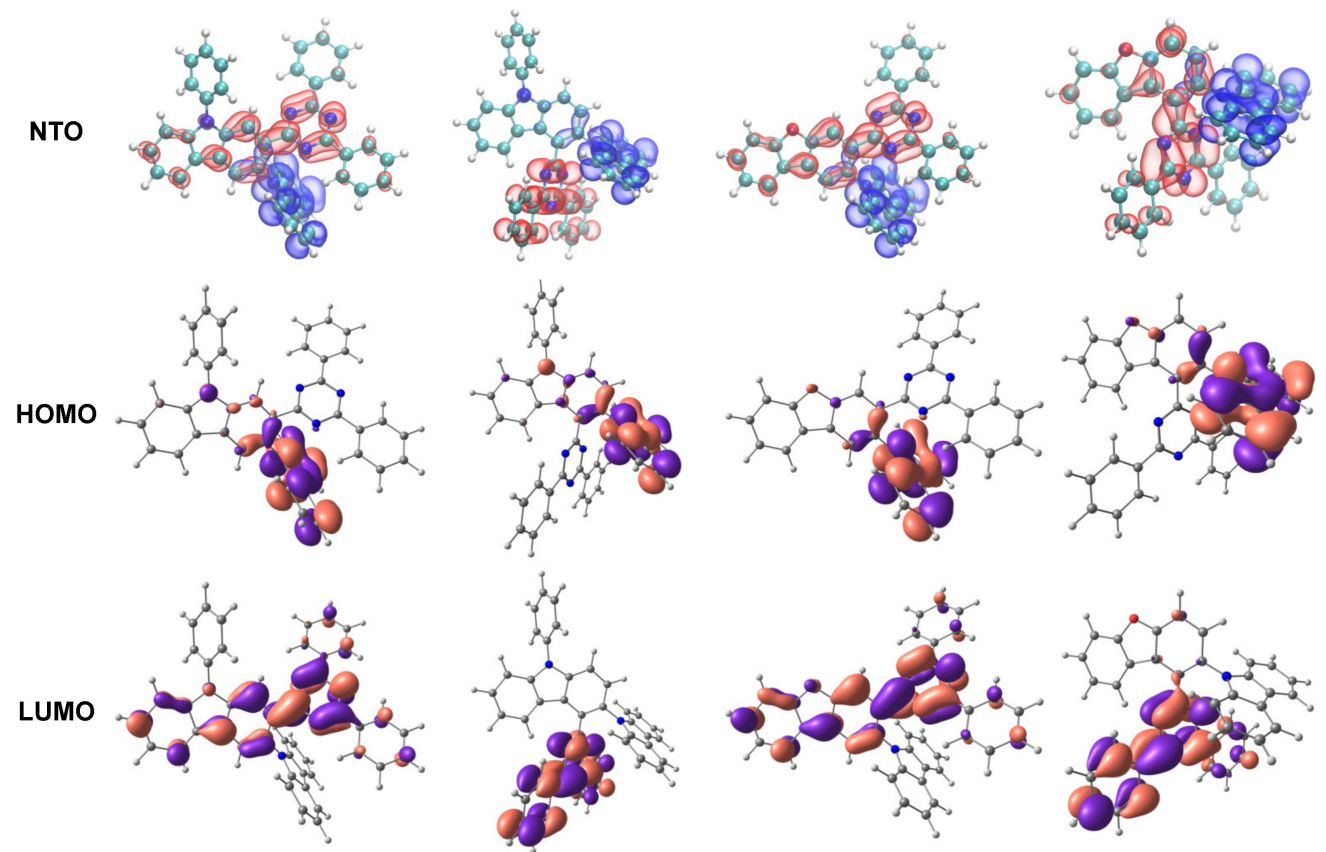

Figure 1. Chemical structures and dihedral angle, natural transition orbital (NTO) hole (blue) and acceptor (red), and HOMO and LUMO orbital distributions.

Table 1. Density functional theory (DFT) calculation data of the thermally activated delayed fluorescence (TADF) emitters.

\begin{tabular}{|c|c|c|c|c|c|c|c|c|}
\hline \multirow[b]{2}{*}{ Name } & \multicolumn{8}{|c|}{ TD-DFT $^{a}$} \\
\hline & $\begin{array}{c}\text { HOMO } \\
(\mathrm{eV})\end{array}$ & $\begin{array}{c}\text { LUMO } \\
(\mathrm{eV})\end{array}$ & $f$ & $\begin{array}{c}S 1 \\
(\mathrm{eV})\end{array}$ & $\begin{array}{c}T 1 \\
(\mathrm{eV})\end{array}$ & $\begin{array}{l}\Delta E_{\mathrm{ST}} \\
(\mathrm{eV})\end{array}$ & $\begin{array}{l}k \text { Reverse Intersystem } \\
\text { Crossing (kRISC) }\left(\mathrm{S}^{-1}\right)\end{array}$ & $\begin{array}{c}\text { Dihedral Angles } \\
\left({ }^{\circ}\right)\end{array}$ \\
\hline BMK-T138 & -5.16 & -1.79 & 0.0067 & 2.77 & 2.67 & 0.10 & 8.17 & 33.6 \\
\hline BMK-T139 & -5.16 & -1.76 & 0.0043 & 2.86 & 2.81 & 0.05 & 2.99 & 58.8 \\
\hline BMK-T317 & -5.30 & -1.94 & 0.0051 & 2.76 & 2.70 & 0.06 & $5.25 \times 10^{5}$ & 35.1 \\
\hline BMK-T318 & -5.31 & -1.88 & 0.0063 & 2.84 & 2.78 & 0.06 & $4.49 \times 10^{5}$ & 53.6 \\
\hline
\end{tabular}

Typical TADF emitter design requires proper placement of electron donor and acceptor moieties, which are connected in a twisted geometry (Figure 2a) to minimize the HOMO-LUMO overlap so that the emission efficiency can be maximized because of the lower S1-T1 energy gap $\left(\Delta E_{\mathrm{ST}}\right)[1,2]$.

All four $\mathrm{C}-\mathrm{C}$ bond TADF emitters were predicted to exhibit properly separated HOMOs and LUMOs as well as lower-energy gaps $\left(\Delta E_{\mathrm{ST}}\right)$ than $0.10 \mathrm{eV}$, owing to the steric hindrance. It was confirmed that the $\Delta E_{\mathrm{ST}}$ values of emitters having a $C-C$ bond connection (about $0.05-0.1 \mathrm{eV}$ ) were three times smaller than those of emitters having $\mathrm{C}-\mathrm{N}$ bond connection $(0.32 \mathrm{eV})$, as reported in the case of compound CPT2 in Adachi's study [28]. Owing to the steric hindrance in our suggested structure, the dihedral angles of BMK-T139 and BMK-T318 were calculated to be $58.8^{\circ}$ and $53.6^{\circ}$, 
respectively, which is much larger than those of $\mathrm{C}-\mathrm{N}$ bond connecting emitters (that of CPT2 was $2.0^{\circ}$ ). The $\Delta E_{\mathrm{ST}}$ values were also reduced from those of the $\mathrm{C}-\mathrm{N}$ bond connecting emitters. On the other hand, the oscillator strength $(f)$ value decreased significantly. Thus, the PL quantum yield (PLQY) was reduced by the influence of the reduced oscillator strength $(f)$. To secure a desirable PLQY, we changed the core structure from carbazole to dibenzofuran.

(a)

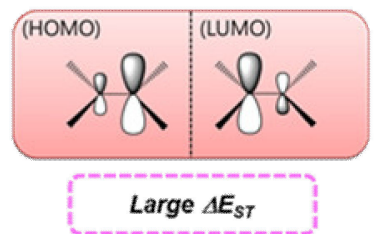

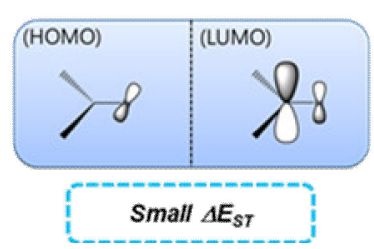

(b)

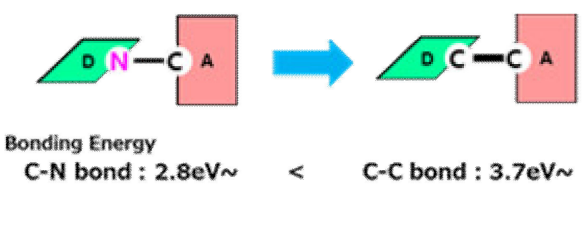

Figure 2. (a) Design principle for $\Delta E_{\mathrm{ST}}$ in orbital geometry. (b) A diagram of bonding energy of $\mathrm{C}-\mathrm{N}$ and $\mathrm{C}-\mathrm{C}$ bonds.

Our emitter design was guided by quantum chemical consideration using time-dependent density functional theory (TD-DFT) calculations, which provided estimates of the $\Delta E_{\mathrm{ST}}$ for the donor-acceptor-based emitters. We performed the TD-DFT calculations on ground-state geometries using the B3LYP [31] functional and the 6-31G* basis set in gas phase. Excited-state geometries and energies were derived by TD-DFT at the MPW1B95 [32]/6-31G(d,p) level of theory. The RISC rate, $k_{R I S C}$, was computed following the Fermi golden rule [33] and Marcus theory [34,35]:

$$
k_{R I S C}=\frac{2 \pi}{\hbar}\left|\left\langle S_{1}\left|H_{S O}\right| T_{1}\right\rangle\right|^{2} \sqrt{\frac{1}{4 \pi k_{B} T \lambda_{S}}} \exp \left[-\frac{\left(\Delta E_{S T}+\lambda_{S}\right)^{2}}{4 \lambda_{S} k_{B} T}\right],
$$

where $\left|\left\langle S_{1}\left|H_{S O}\right| T_{1}\right\rangle\right|$ is the spin-orbit coupling (SOC) matrix element between the $S_{1}$ and $T_{1}$ states. $k_{B}$ is the Boltzmann constant, and $T$ is temperature, which was set to $298 \mathrm{~K}$. $\Delta E_{S T}$ denotes the energy difference between the $S_{1}$ and $T_{1}$ states, and $\lambda_{S}$ represents the reorganization energy, which can be formulated as

$$
\lambda_{S}=E_{S 1}\left(T_{1} \text { geometry }\right)-E_{S 1}\left(S_{1} \text { geometry }\right)
$$

All DFT and TD-DFT computations were performed using the Gaussian 09 program, and the SOC calculations were performed using the Amsterdam Density Functional (ADF). The SOC and reorganization energy values are summarized in the supporting information (Table S1).

Based on this calculation, emitters possessing the $\mathrm{C}-\mathrm{C}$ bond connections were highly advantageous in terms of material stability because their bonding energies increased. The results indicate that the calculated bonding energy values of $\mathrm{C}-\mathrm{N}$ and $\mathrm{C}-\mathrm{C}$ were 2.8 and $3.7 \mathrm{eV}$, respectively (Figure $2 \mathrm{~b}$ ). Among the four suggested compounds, both BMK-T317 and BMK-T318, having a dibenzofuran backbone, were predicted to have much higher values of kRISC than those of BMK-T138 and BMK-T139, having a carbazole backbone (Table 1). This indicates that BMK-T317 and BMK-T318 had a delayed fluorescence lifetime $\left(\tau_{\mathrm{DF}}\right)$ with a high quantum yield.

\subsection{Photophysical Properties}

Optical absorption and PL spectra of the BMK-T138, BMK-T139, BMK-T 317, and BMK-T318 in dilute toluene solution $\left(1 \times 10^{-4} \mathrm{M}\right)$ are shown in Figure S2 and S3. The key values of the photophysical properties of the four blue TADF emitters (i.e., PL emission maximum $\left(\lambda_{\max }{ }^{\mathrm{em}}\right), E_{\mathrm{S}}, E_{\mathrm{T}}, \Delta E_{\mathrm{ST}}$, full width at half-maximum (FWHM), and PLQY) are summarized in Table 2. The PL emission maxima $\left(\lambda_{\max }{ }^{\mathrm{em}}\right)$ of BMK-T138, BMK-T139, BMK-T 317, and BMK-T319 were observed at 478, 480, 469, and $466 \mathrm{~nm}$, respectively. All TADF emitters exhibited narrow FWHM of $66-75 \mathrm{~nm}$. To calculate the $\Delta E_{\mathrm{ST}}$ of the TADF emitters, we compared the $E_{\mathrm{T}}$, which was measured at $77 \mathrm{~K}$, and the $E_{\mathrm{S}}$, which was measured at room temperature, from the onset energy of the fluorescence and phosphorescence spectra. 
The measured HOMO-LUMO energy levels of the TADF emitters are summarized in the supporting information (Table S2).

Table 2. Physical properties of the TADF emitters in the solution state.

\begin{tabular}{|c|c|c|c|c|c|c|c|c|}
\hline \multirow[b]{2}{*}{ Name } & \multicolumn{7}{|c|}{ Physical Properties of TADF Emitters ${ }^{\text {a }}$} & \multirow[b]{2}{*}{$\begin{array}{c}\text { kRISC } \\
(\%)\end{array}$} \\
\hline & $\begin{array}{l}\text { Photoluminescence } \\
\text { (PL) (nm) }\end{array}$ & $\begin{array}{l}\text { Full width at Half-Maximum } \\
\text { (FWHM) (nm) }\end{array}$ & $\begin{array}{c}E_{\mathrm{S}} \\
(\mathrm{eV})^{\mathrm{b}}\end{array}$ & $\begin{array}{c}E_{\mathrm{T}} \\
(\mathrm{eV})^{\mathrm{b}}\end{array}$ & $\begin{array}{l}\Delta E_{\mathrm{ST}} \\
(\mathrm{eV})^{\mathrm{c}}\end{array}$ & $\begin{array}{l}\text { PL Quantum } \\
\text { Yield (PLQY) }^{d}\end{array}$ & $\begin{array}{c}\tau_{\mathrm{DF}}{ }^{\mathrm{d}} \\
(\mu \mathrm{s})\end{array}$ & \\
\hline BMK-T138 & 478 & 66 & 2.87 & 2.82 & 0.05 & 0.741 & 163 & 32.10 \\
\hline BMK-T139 & 480 & 72 & 2.87 & 2.75 & 0.12 & 0.622 & 147 & 50.80 \\
\hline BMK-T317 & 469 & 72 & 2.82 & 2.81 & 0.01 & 0.697 & 51 & 60.53 \\
\hline BMK-T318 & 466 & 75 & 2.79 & 2.90 & -0.11 & 0.737 & 21 & 63.09 \\
\hline
\end{tabular}

${ }^{a}$ Measured in toluene solution. ${ }^{b}$ Energy of the singlet and triplet estimated from onset of wavelength in toluene solution. ${ }^{\mathrm{c}} \Delta E_{\mathrm{ST}}=E_{\mathrm{S}}-E_{\mathrm{T}} .{ }^{\mathrm{d}} \mathrm{PLQY}$ values were estimated with DPEPO host.

The $E_{\mathrm{S}} / E_{\mathrm{T}} / \Delta E_{\mathrm{ST}}$ values of the BMK-T138 and BMK-T139 emitters were 2.87/2.82/0.05 eV and $2.87 / 2.75 / 0.12 \mathrm{eV}$, respectively, from the onset energy of the fluorescence and phosphorescence spectra. On the other hand, BMK-T317 and BMK-T318, which used dibenzofuran-based emitters, exhibited smaller $\Delta E_{\mathrm{ST}}$ values, resulting from the $E_{\mathrm{S}}$ decrease and the $E_{\mathrm{T}}$ increase. As expected from the DFT calculation, the singlet-triplet energy gaps of the emitters having $\mathrm{C}-\mathrm{C}$ bonds were lower than that of the CPT2 having a C-N bond (that of CPT2 was $0.32 \mathrm{eV}$ ) [28].

The PLQYs of BMK-T138, BMK-T139, BMK-T 317, and BMK-T318 were found to be $0.741,0.622$, 0.697 , and 0.737 , respectively. To improve the RISC yield while maintaining efficient PLQY of TADF emitters, we modified the core structure from a carbazole core to a dibenzofuran core.

Using the PLQY and transient PL data, the RISC yield was calculated for each emitter. Because the $\tau_{\mathrm{DF}}$ values of the dibenzofuran-based TADF emitters decreased as expected in the calculation, the RISC yields of the dibenzofuran-based TADF emitters were shown to be higher than those of the carbazole-based TADF emitters (63.09 vs. 32.10). Additionally, large distortions of the donor from the acceptor linker increased the RISC yield with a reduced $\tau_{\mathrm{DF}}[36]$.

\subsection{Device Performances}

To compare the $\mathrm{C}-\mathrm{N}$ and $\mathrm{C}-\mathrm{C}$ bond skeleton molecules, device evaluation was conducted using a DPEPO host for CPT2 and BMK-T138 TADF emitters. Although CPT2 exhibited short delayed fluorescence lifetimes $\left(\tau_{\mathrm{DF}}\right)$, owing to its linear-type TADF material, it showed very efficient roll-off because of the low bond dissociation energy of the $\mathrm{C}-\mathrm{N}$ bond as described in Figure $2 \mathrm{~b}$. Despite the long $\tau_{\mathrm{DF}}$ value of BMK-T138 $(163 \mu \mathrm{s})$, the efficiency roll-off was significantly reduced because of the large PLQY and C-C bond stability. Furthermore, the maximum external quantum efficiency of BMK-T138 was 19.6\%, and the current efficiency (CE) was $40.53 \mathrm{~cd} / \mathrm{A}$, respectively. The current density-voltage-luminance (J-V-L), and quantum efficiency-luminance-current efficiency characteristics of TADF devices with $\mathrm{C}-\mathrm{C}$ bond skeleton emitters are shown in Figure 3.

To summarize, the performances of the devices containing the $\mathrm{C}-\mathrm{C}$ bond skeleton molecules appeared to have had the greatest effect on the stability of the material with a high PQLY $(74 \%)$. Comparison of the physical properties of devices containing either a $\mathrm{C}-\mathrm{C}$ bond emitter (BMK-T138) or a $\mathrm{C}-\mathrm{N}$ bond emitter (CPT2) in a DPEPO host is summarized in Table 3. The advantage of the emitter equipped with a $\mathrm{C}-\mathrm{C}$ bond skeleton is the increased overlap density of the HOMO/LUMO distribution with greater dihedral angle between donor and acceptor (Figure S4). By employing the dibenzofuran moiety without a phenyl linker as a core structure and increasing the overall rigidity and overlap density, we achieved short delayed fluorescence lifetimes $\left(\tau_{\mathrm{DF}}\right)$. Moreover, the rigid structure was expected to result in reduced nonradiative mechanisms during light emission. 


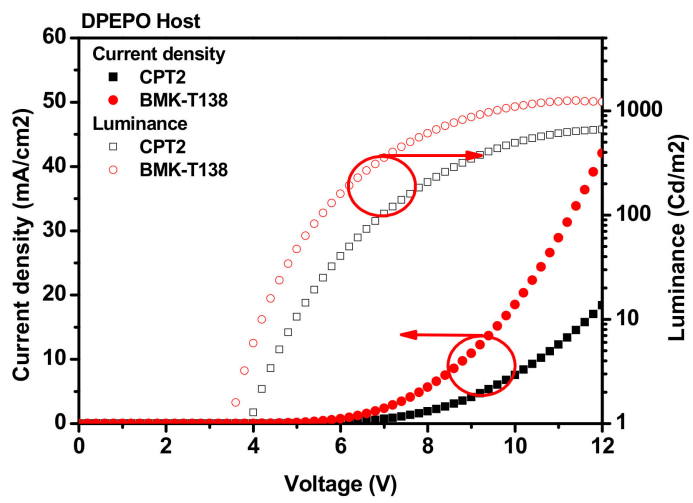

(a)

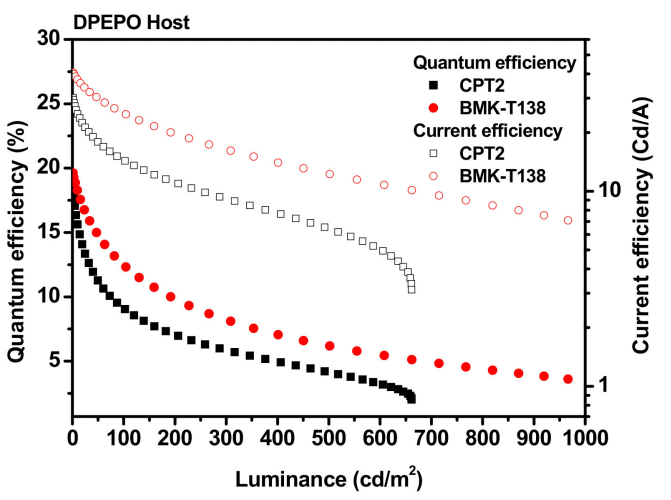

(b)

Figure 3. CPT2 and BMK-T138 TADF devices with DPEPO (15\%) host: (a) current density-voltage-luminance (J-V-L); (b) External quantum efficiency (EQE) and current efficiency (CE) versus current density curves.

Table 3. Device performance comparison between a $\mathrm{C}-\mathrm{C}$ bond emitter (BMK-T138) and a $\mathrm{C}-\mathrm{N}$ bond emitter (CPT2) in the DPEPO host.

\begin{tabular}{|c|c|c|c|c|c|c|c|}
\hline Name & $\mathrm{EQE}_{\max }(\%)$ & $\mathrm{EQE}^{\mathrm{a}}(\%)$ & $\mathrm{CE}_{\max }(\mathrm{Cd} / \mathrm{A})$ & Roll-off (\%) & EL (nm) & $\mathrm{V}_{\mathrm{d}}(\mathrm{V})$ & Color Coordinates \\
\hline СРT2 & 18.7 & 4.1 & 30.1 & 78.7 & 470 & 10.06 & $0.162,0.206$ \\
\hline BMK-T138 & 19.6 & 6.2 & 40.6 & 69.8 & 480 & 7.59 & $0.155,0.314$ \\
\hline
\end{tabular}

Compared to the $\mathrm{C}-\mathrm{N}$ bonding blue TADF emitter, those containing a $\mathrm{C}-\mathrm{C}$ bond skeleton were expected to secure a high efficiency with great stability for the device. As expected, the BMK-T138 TADF emitter with a long $\tau_{\mathrm{DF}}$ significantly improved the external quantum efficiency for the DPEPO host system. Thus, the device's material stability was an important factor for improving device performances. Therefore, we conducted additional photophysical experiments using the mCBP-CN host instead of DPEPO [37]. Although TADF emitters with a DPEPO host are characteristically adopted for device performance tests, we examined our emitters using the $\mathrm{mCBP}-\mathrm{CN}$ host, focusing on optimizing the devices with suppressed efficiency roll-off [38]. DPEPO has the advantage of high efficiency with a wide band gap and high triplet energy to boost high-efficiency TADF devices. However, it is not suitable for device stability because it is an unstable ET type host material with low stability in EML. Because DPEPO has a quite shallow LUMO for an ET-type host, all TADF emitters doped in DPEPO have deeper LUMOs than that of DPEPO. When all TADF emitters with DPEPO host have a large band gap, both hole and electron carriers are likely to be trapped inside the transport layer. As a result, these trapped carriers expose dopant molecules under electrical stress, rendering the host-dopant system less efficient.

Because mCBP-CN has an ET-type character with deep LUMO, the emission layer composed of the mCBP-CN host and TADF emitters showed better charge transport and balance than that of the DPEPO host. Therefore, we employed $\mathrm{mCBP}-\mathrm{CN}$, which is well fitted to our blue TADF emitters, having shallow LUMOs and HOMOs. Generally, the deep HOMO energy level causes a low hole injection rate in the hole-only devices (HODs). The TADF emitters having deep HOMO energy levels decreased the HOD strength. The four TADF molecules have similar tendencies, as shown in Figure S5b. Although the dibenzofuran core structure changed the energy level, it provided a highly efficient performance by minimizing the charge balance in the emission layer for TADF devices. Figure S5b shows the correlation between the hole current and HOMO energy levels.

TADF devices having dibenzofuran-based emitters enhanced device performance (BMK-T317 and BMK-T318) compared to those containing carbazole-based emitters, as shown in Figure 4 and Table 4. Dibenzofuran-based devices showed a significantly reduced turn-on voltage of 4.68 and $4.36 \mathrm{~V}$, exhibiting increased CEs of 37.6 and $38.2 \mathrm{~cd} / \mathrm{A}$, respectively. On the other hand, carbazole-based 
emitters showed high turn-on voltages (5.16 and 5.71 V) and CEs of 33.8 and $21.8 \mathrm{~cd} / \mathrm{A}$, respectively. BMK-T317 and BMK-T318 also exhibited enhanced device performance, showing an EQE of 19.9\% and $18.8 \%$, respectively. In addition, the maximum luminance of BMK-T318 was much lower than that of all TADF devices, presumably owing to the low current density. BMK-T318 showed the lowest current density, and BMK-T139 showed the highest. This can be correlated with the energy levels of the core structures. The difference between the HOMO energy level of BMK-T318 and that of the mCBP-CN host was only $0.39 \mathrm{eV}$, whereas the HOMO energy level difference between BMK-T138 and mCBP-CN host was $0.50 \mathrm{eV}$. Therefore, BMK-T318 is suitable for the blue TADF because the energy barrier of BMK-T318 was the lowest.
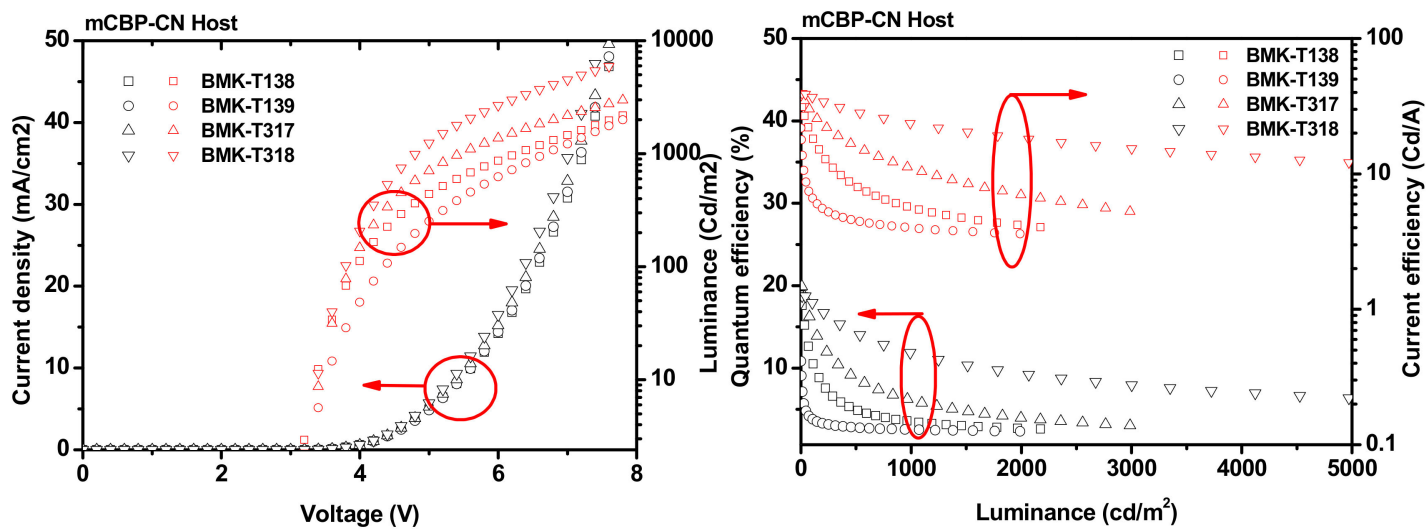

Figure 4. BMK-T138, BMK-T139, BMK-T317, and BMK-T318 TADF devices with an mCBP-CN (15\%) host: (a) current $(J-V-L)$; (b) EQE and CE versus current density curves.

Table 4. Device performance of the TADF emitters having an $\mathrm{mCBP}-\mathrm{CN}$ host.

\begin{tabular}{|c|c|c|c|c|c|c|c|c|}
\hline TADFE mitter & $\mathrm{EQE}_{\max }(\%)$ & $\mathrm{EQE}^{\mathrm{a}}(\%)$ & $\mathrm{CE}_{\max }(\mathrm{Cd} / \mathrm{A})$ & Roll-off (\%) & $\mathrm{EL}(\mathrm{nm})$ & $J^{\mathrm{b}}\left(\mathrm{mA} / \mathrm{cm}^{2}\right)$ & $V_{\mathrm{d}}{ }^{\mathrm{a}}(\mathrm{V})$ & Color Coordinates \\
\hline BMK-T138 & 18.8 & 5.0 & 33.9 & 75.8 & 473 & 8.17 & 5.16 & $0.141,0.240$ \\
\hline BMK-T139 & 10.8 & 2.8 & 21.8 & 79.3 & 473 & 11.08 & 5.71 & $0.160,0.321$ \\
\hline BMK-T317 & 19.9 & 8.8 & 37.6 & 57.3 & 475 & 3.15 & 4.68 & $0.147,0.228$ \\
\hline BMK-T318 & 18.8 & 14.3 & 38.2 & 26.7 & 481 & 1.75 & 4.36 & $0.152,0.274$ \\
\hline
\end{tabular}

${ }^{\mathrm{a}}$ EQE at luminance of $500 \mathrm{~cd} / \mathrm{m}^{2},{ }^{\mathrm{b}}$ Current density $(J)$ at driving voltage of $5 \mathrm{~V}$.

Dibenzofuran-based materials also showed significantly enhanced efficiency roll-off because of the relatively low $\tau_{\mathrm{DF}}$ values of BMK-T317 and BMK-T318 (51 and $21 \mu \mathrm{s}$, respectively), compared to the other $\tau_{\mathrm{DF}}$ values of carbazole-based materials $\left(\mathrm{BMK}-\mathrm{T} 138, \tau_{\mathrm{DF}}=163 \mu \mathrm{s}\right.$ and BMK-T139, $\left.\tau_{\mathrm{DF}}=147 \mu \mathrm{s}\right)$. In the case of BMK-T138, a large $\tau_{\mathrm{DF}}$ value led to relatively poor efficiency roll-off than those of other $\mathrm{C}-\mathrm{C}$ bond emitters. This implies that facilitating efficient host exciton transport from the recombination zone to the ground state with a high RISC yield is an important factor for securing TADF device stability. We note that RISC should be much faster than nonradioactive emission to the ground state to efficiently transfer energy from triplet to singlet. Therefore, device stability can be improved, and it can be advantageous in terms of device lifetime.

Electroluminescence (EL) spectra of all devices were investigated, as shown in Table 4. The EL emission peaks were located at 473, 473, 475, and $481 \mathrm{~nm}$, respectively (Table 4 and Figure 5). The EL emission peaks of compounds possessing the dibenzofuran backbone group were a bit red-shifted from the PL peak values. 


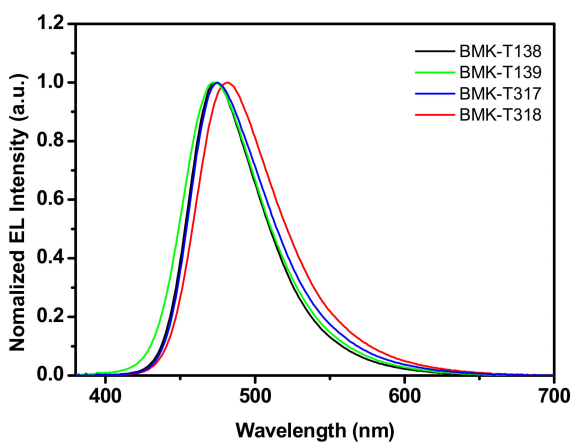

Figure 5. The EL spectra of BMK-T138, BMK-T139, BMK-T317, and BMK-T318 TADF devices.

\section{Conclusions}

A new design strategy of TADF emitters was proposed, entailing interlocking a donor with an acceptor through a $\mathrm{C}-\mathrm{C}$ bond. The photophysical property data obtained from the synthesized compounds provided important insights on increasing stability of TADF materials during the photoluminescence process and the possibility of blue emission. A series of C-C-bond TADF emitters were introduced, featuring large dihedral angle of the central core in adjacent donor and acceptor arrangement provoking large steric hindrance. These emitters exhibited properly separated HOMO-LUMO overlap and low $\Delta E_{\mathrm{ST}}$ calculation values $(<0.12 \mathrm{eV})$. The photophysical properties of $\mathrm{C}-\mathrm{C}$ bonding TADF emitters were characterized by a narrow FWHM of $66 \mathrm{~nm}$ and a blue emission of $466 \mathrm{~nm}$ with a short fluorescence lifetime $\left(\tau_{\mathrm{DF}}\right)$ of $21 \mu \mathrm{s}$ in a solution state. The EL experimental results indicated a desirable EL peak of $473 \mathrm{~nm}$ and a high external quantum efficiency value of $19.9 \%$. Especially clear reduction of the efficiency roll-off characteristics (26.7\%) was observed in the case of dibenzofuran core structure. It is evident that the efficiency roll-off affects the operational stability of TADF emitters. Therefore, the TADF emitters having a $\mathrm{C}-\mathrm{C}$ bond connection have the potential to secure a high efficiency and long life.

Supplementary Materials: The following are available online at http://www.mdpi.com/2079-4991/9/12/1735/s1, Figure S1: (a) TGA and (b) DSC curves of TADF emitters, Figure S2: Optical absorption and photoluminescence spectra of (a) BMK-T138 and (b) BMK-T139, Figure S3: Optical absorption and photoluminescence spectra of (a) BMK-T317 and (b) BMK-T318, Figure S4: Overlap density-delayed fluorescence lifetimes $\left(\tau_{D F}\right)$ data, Table S1: Additional theoretical computations data of the TADF emitters, Table S2: Measured HOMO-LUMO energy levels of the TADF emitters.

Author Contributions: T.H.K., and S.O.J. conceived the study; T.H.K., S.O.J., M.N., and Y.S.C. synthesized and characterized the materials; S.O.J., and M.N. designed the molecules; J.S.K., and M.S. fabricated and tested devices; S.-G.I. performed steady-state and transient PL study; H.L. performed calculations and analyzed theoretical predictions; T.H.K., and S.O.J. wrote the first version of the manuscript; All authors contributed to discussion on the study and editing the manuscript; B.M.K., and S.K. organized and supervised the project.

Funding: This research received no external funding.

Acknowledgments: This material is based upon work supported in part by Samsung Advanced Institute of Technology (SAIT). Tae Hui Kwon and Soon Ok Jeon contributed equally to this work.

Conflicts of Interest: The authors declare no conflicts of interest.

\section{References}

1. Kido, J.; Kimura, M.; Nagai, K. Multilayer white light-emitting organic electroluminescent device. Science 1995, 27, 1332-1334. [CrossRef] [PubMed]

2. Sun, Y.; Giebink, N.C.; Kanno, H.; Ma, B.; Thompson, M.E.; Forrest, S.R. Management of singlet and triplet excitons for efficient white organic light-emitting devices. Nature 2006, 440, 908-912. [CrossRef] [PubMed]

3. Reineke, S.; Lindner, F.; Schwartz, G.; Seidler, N.; Walzer, K.; Lussem, B.; Leo, K. White organic light-emitting diodes with fluorescent tube efficiency. Nature 2009, 459, 234-238. [CrossRef] [PubMed] 
4. Han, T.-H.; Lee, Y.; Choi, M.-R.; Woo, S.-H.; Bae, S.-H.; Hong, B.H.; Ahn, J.-H.; Lee, T.-W. Extremely efficient flexible organic light-emitting diodes with modified graphene anode. Nat. Photonics 2012, 6, 105-110. [CrossRef]

5. Chen, T.; Zheng, L.; Yuan, J.; An, Z.; Chen, R.; Tao, Y.; Li, H.; Xie, X.; Huang, W. Understanding the Control of Singlet-Triplet Splitting for Organic Exciton Manipulating: A Combined Theoretical and Experimental Approach. Sci. Rep. 2015, 5, 10923. [CrossRef] [PubMed]

6. Komatsu, R.; Ohsawa, T.; Sasabe, H.; Nakao, K.; Hayasaka, Y.; Kido, J. Manipulating the Electronic Excited State Energies of Pyrimidine-Based Thermally Activated Delayed Fluorescence Emitters to Realize Efficient Deep-Blue Emission. ACS Appl. Mater. Interfaces 2017, 9, 4742-4749. [CrossRef]

7. Sato, K.; Shizu, K.; Yoshimura, K.; Kawada, A.; Miyazaki, H.; Adachi, C. Organic luminescent molecule with energetically equivalent singlet and triplet excited states for organic light-emitting diodes. Phys. Rev. Lett. 2013, 110, 247401. [CrossRef]

8. Volz, D. Review of organic light-emitting diodes with thermally activated delayed fluorescence emitters for energy-efficient sustainable light sources and displays. J. Photonics Energy 2016, 6, 020901. [CrossRef]

9. Yang, Z.; Mao, Z.; Xie, Z.; Zhang, Y.; Liu, S.; Zhao, J.; Xu, J.; Chi, Z.; Aldred, M.P. Recent advances in organic thermally activated delayed fluorescence materials. Chem. Soc. Rev. 2017, 46, 915-1016. [CrossRef]

10. Uoyama, H.; Goushi, K.; Shizu, K.; Nomura, H.; Adachi, C. Highly efficient organic light-emitting diodes from delayed fluorescence. Nature 2012, 492, 234-238. [CrossRef]

11. Tao, Y.; Yuan, K.; Chen, T.; Xu, P.; Li, H.; Chen, R.; Zheng, C.; Zhang, L.; Huang, W. Thermally activated delayed fluorescence materials towards the breakthrough of organoelectronics. Adv. Mater. 2014, 26, 7931-7958. [CrossRef]

12. Zhang, Q.; Li, B.; Huang, S.; Nomura, H.; Tanaka, H.; Adachi, C. Efficient blue organic light-emitting diodes employing thermally activated delayed fluorescence. Nat. Photonics 2014, 8, 326-332. [CrossRef]

13. Hirata, S.; Sakai, Y.; Masui, K.; Tanaka, H.; Lee, S.Y.; Nomura, H.; Nakamura, N.; Yasumatsu, M.; Nakanotani, H.; Zhang, Q.; et al. Highly efficient blue electroluminescence based on thermally activated delayed fluorescence. Nat. Mater. 2015, 14, 330-336. [CrossRef] [PubMed]

14. Zhang, Q.; Tsang, D.; Kuwabara, H.; Hatae, Y.; Li, B.; Takahashi, T.; Lee, S.Y.; Yasuda, T.; Adachi, C. Nearly $100 \%$ internal quantum efficiency in undoped electroluminescent devices employing pure organic emitters. Adv. Mater. 2015, 27, 2096-2100. [CrossRef] [PubMed]

15. Kim, M.; Jeon, S.K.; Hwang, S.H.; Lee, J.Y. Stable blue thermally activated delayed fluorescent organic light-emitting diodes with three times longer lifetime than phosphorescent organic light-emitting diodes. Adv. Mater. 2015, 27, 2515-2520. [CrossRef] [PubMed]

16. Sun, J.W.; Baek, J.Y.; Kim, K.-H.; Moon, C.-K.; Lee, J.-H.; Kwon, S.-K.; Kim, Y.-H.; Kim, J.-J. Thermally Activated Delayed Fluorescence from Azasiline Based Intramolecular Charge-Transfer Emitter (DTPDDA) and a Highly Efficient Blue Light Emitting Diode. Chem. Mater. 2015, 27, 6675-6681. [CrossRef]

17. Youn Lee, S.; Yasuda, T.; Nomura, H.; Adachi, C. High-efficiency organic light-emitting diodes utilizing thermally activated delayed fluorescence from triazine-based donor-acceptor hybrid molecules. Appl. Phys. Lett. 2012, 101, 093306. [CrossRef]

18. Rajamalli, P.; Senthilkumar, N.; Gandeepan, P.; Ren-Wu, C.C.; Lin, H.W.; Cheng, C.H. Method for Reducing the Singlet-Triplet Energy Gaps of TADF Materials for Improving the Blue OLED Efficiency. ACS Appl. Mater. Interfaces 2016, 8, 27026-27034. [CrossRef]

19. Yang, Y.; Wang, S.; Zhu, Y.; Wang, Y.; Zhan, H.; Cheng, Y. Thermally Activated Delayed Fluorescence Conjugated Polymers with Backbone-Donor/Pendant-Acceptor Architecture for Nondoped OLEDs with High External Quantum Efficiency and Low Roll-Off. Adv. Funct. Mater. 2018, 28, 1706916. [CrossRef]

20. Park, Y.-S.; Lee, S.; Kim, K.-H.; Kim, S.-Y.; Lee, J.-H.; Kim, J.-J. Exciplex-Forming Co-host for Organic Light-Emitting Diodes with Ultimate Efficiency. Adv. Funct. Mater. 2013, 23, 4914-4920. [CrossRef]

21. Cho, Y.J.; Chin, B.D.; Jeon, S.K.; Lee, J.Y. 20\% External Quantum Efficiency in Solution-Processed Blue Thermally Activated Delayed Fluorescent Devices. Adv. Funct. Mater. 2015, 25, 6786-6792. [CrossRef]

22. Liu, W.; Zheng, C.J.; Wang, K.; Chen, Z.; Chen, D.Y.; Li, F.; Ou, X.M.; Dong, Y.P.; Zhang, X.H. Novel Carbazol-Pyridine-Carbonitrile Derivative as Excellent Blue Thermally Activated Delayed Fluorescence Emitter for Highly Efficient Organic Light-Emitting Devices. ACS Appl. Mater. Interfaces 2015, 7, 18930-18936. [CrossRef] [PubMed] 
23. Cho, Y.J.; Jeon, S.K.; Lee, S.-S.; Yu, E.; Lee, J.Y. Donor Interlocked Molecular Design for Fluorescence-like Narrow Emission in Deep Blue Thermally Activated Delayed Fluorescent Emitters. Chem. Mater. 2016, 28, 5400-5405. [CrossRef]

24. Kang, J.S.; Hong, T.R.; Kim, H.J.; Son, Y.H.; Lampande, R.; Kang, B.Y.; Lee, C.; Bin, J.-K.; Lee, B.S.; Yang, J.H.; et al. High-performance bipolar host materials for blue TADF devices with excellent external quantum efficiencies. J. Mater. Chem. C 2016, 4, 4512-4520. [CrossRef]

25. Lee, Y.H.; Park, S.; Oh, J.; Shin, J.W.; Jung, J.; Yoo, S.; Lee, M.H. Rigidity-Induced Delayed Fluorescence by Ortho Donor-Appended Triarylboron Compounds: Record-High Efficiency in Pure Blue Fluorescent Organic Light-Emitting Diodes. ACS Appl. Mater. Interfaces 2017, 9, 24035-24042. [CrossRef]

26. Rajamalli, P.; Thangaraji, V.; Senthilkumar, N.; Ren-Wu, C.-C.; Lin, H.-W.; Cheng, C.-H. Thermally activated delayed fluorescence emitters with a $m, m$-di-tert-butyl-carbazolyl benzoylpyridine core achieving extremely high blue electroluminescence efficiencies. J. Mater. Chem. C 2017, 5, 2919-2926. [CrossRef]

27. Zhang, D.; Song, X.; Cai, M.; Kaji, H.; Duan, L. Indolocarbazole-Isomer Derivatives as Highly Emissive Emitters and Ideal Hosts for Thermally Activated Delayed Fluorescent OLEDs with Alleviated Efficiency Roll-Off. Adv. Mater. 2018, 30. [CrossRef]

28. Chen, X.K.; Tsuchiya, Y.; Ishikawa, Y.; Zhong, C.; Adachi, C.; Bredas, J.L. A New Design Strategy for Efficient Thermally Activated Delayed Fluorescence Organic Emitters: From Twisted to Planar Structures. Adv. Mater. 2017, 29. [CrossRef]

29. Fukagawa, H.; Shimizu, T.; Iwasaki, Y.; Yamamoto, T. Operational lifetimes of organic light-emitting diodes dominated by Forster resonance energy transfer. Sci. Rep. 2017, 7, 1735. [CrossRef]

30. Ward, J.S.; Nobuyasu, R.S.; Batsanov, A.S.; Data, P.; Monkman, A.P.; Dias, F.B.; Bryce, M.R. The interplay of thermally activated delayed fluorescence (TADF) and room temperature organic phosphorescence in sterically-constrained donor-acceptor charge-transfer molecules. Chem. Commun. (Camb.) 2016, 52, 2612-2615. [CrossRef] [PubMed]

31. Stephens, P.J.; Devlin, F.J.; Chabalowski, C.F.; Frisch, M.J. Ab Initio calculation of vibrational absorption and circular dichroism spectra using density functional force fields. J. Phys. Chem. 1994, 98, 11623-11627. [CrossRef]

32. Zhao, Y.; Truhlar, D.G. Hybrid meta density functional theory methods for thermochemistry, thermochemical kinetics, and noncovalent interactions: the MPW1B95 and MPWB1K models and comparative assessments for hydrogen bonding and van der Waals interactions. J. Phys. Chem. A 2004, 108, 6908-6918. [CrossRef]

33. Samanta, P.K.; Kim, D.; Coropceanu, V.; Bredas, J.L. Up-Conversion Intersystem Crossing Rates in Organic Emitters for Thermally Activated Delayed Fluorescence: Impact of the Nature of Singlet vs Triplet Excited States. J. Am. Chem. Soc. 2017, 139, 4042-4051. [CrossRef] [PubMed]

34. Marcus, R.A. On the theory of oxidation-reduction reactions involving electron transfer. I. J. Chem. Phys. 1956, 24, 966-978. [CrossRef]

35. Feng, S.; Wen, K.; Si, Y.; Guo, X.; Zhang, J. Theoretical studies on thermally activated delayed fluorescence mechanism of a series of organic light-emitting diodes emitters comprising 2,7-diphenylamino-9,9-dimethylacridine as electron donor. J. Comput. Chem. 2018, 39, 2601-2606. [CrossRef]

36. Yu, J.G.; Han, S.H.; Lee, H.L.; Hong, W.P.; Lee, J.Y. A novel molecular design employing a backbone freezing linker for improved efficiency, sharpened emission and long lifetime in thermally activated delayed fluorescence emitters. J. Mater. Chem. C 2019, 7, 2919-2926. [CrossRef]

37. Cooper, M.W.; Zhang, X.; Zhang, Y.; Jeon, S.O.; Lee, H.; Kim, S.; Fuentes-Hernandez, C.; Barlow, S.; Kippelen, B.; Marder, S.R. Effect of the Number and Substitution Pattern of Carbazole Donors on the Singlet and Triplet State Energies in a Series of Carbazole-Oxadiazole Derivatives Exhibiting Thermally Activated Delayed Fluorescence. Chem. Mater. 2018, 30, 6389-6399. [CrossRef]

38. Ihn, S.G.; Lee, N.; Jeon, S.O.; Sim, M.; Kang, H.; Jung, Y.; Huh, D.H.; Son, Y.M.; Lee, S.Y.; Numata, M.; et al. An Alternative Host Material for Long-Lifespan Blue Organic Light-Emitting Diodes Using Thermally Activated Delayed Fluorescence. Adv. Sci. (Weinh.) 2017, 4, 1600502. [CrossRef] [PubMed]

(C) 2019 by the authors. Licensee MDPI, Basel, Switzerland. This article is an open access article distributed under the terms and conditions of the Creative Commons Attribution (CC BY) license (http://creativecommons.org/licenses/by/4.0/). 